\title{
Parasitismo natural de Spodoptera frugiperda (Smith) (Lepidoptera: Noctuidae), en cuatro departamentos de Paraguay
}

\section{Natural parasitism of Spodoptera frugiperda (Smith) (Lepidoptera: Noctuidae) in four departments in Paraguay}

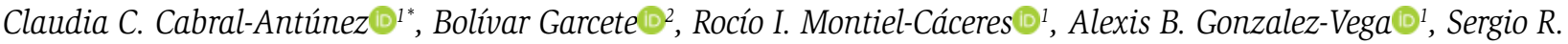 \\ Cárdenas ${ }^{1}$, Nancy Armoa ${ }^{1}$ y María Bernarda Ramírez de López@ ${ }^{1}$
}

*Autor de correspondencia: claudia_c_cabral@hotmail.com

Recibido: 25 de octubre de 2018 - Aceptado: 23 de noviembre de 2018

1 Universidad Nacional de Asunción, Facultad de Ciencias Agrarias, Asunción, Paraguay

2 Universidad Nacional de Asunción, Facultad de Ciencias Exactas y Naturales, Asunción, Paraguay

\begin{abstract}
Palabras clave:
porcentaje de parasitoidismo; fauna de parasitoides; maíz; gusano cogollero
\end{abstract}

\section{Key words:}

percentage parasitism; parasitoid fauna; maize; fall army worm

\section{Resumen}

El impacto que los enemigos naturales producen sobre las plagas de cultivos es un factor importante a tener en cuenta en el momento de evaluar los métodos de control a aplicar. En este contexto, la fauna propia de una región y su influencia sobre una plaga en particular son datos relevantes. Este artículo reporta la ocurrencia de parasitoides asociados de manera natural al gusano cogollero Spodoptera frugiperda (Smith, 1797) y su impacto porcentual calculado en la base de cría de larvas del hospedero recolectados durante el período 2015-2016 en Paraguay en los departamentos de Caaguazú, Alto Paraná, Canindeyú e Itapúa. Los parasitoides registrados fueron: Dissomphalus spp. (Hymenoptera: Bethylidae), Exasticolus fuscicornis Cameron (Hymenoptera: Braconidae), Ophion spp. (Hymenoptera: Ichneumonidae), Archytas spp. (Diptera: Tachinidae) y Winthenia spp. (Diptera: Tachinidae).

\section{Abstract}

The impact produced by natural enemies on culture pests is a very important factor to consider at the moment of evaluating control methods. In this context, the characteristic fauna of a region and its influence on a particular pest are relevant data. This paper records the occurrence of parasitoids naturally associated with the fall army worm Spodoptera frugiperda (Smith, 1797) and their percentual impact calculated from rearing larvae of the host collected during the period 2015 - 2016 in Paraguay in the departments of Caaguazú, Alto Paraná, Canindeyú and Itapúa. The parasitoids recorded were: Dissomphalus spp. (Hymenoptera: Bethylidae), Exasticolus fuscicornis Cameron (Hymenoptera: Braconidae), Ophion spp. (Hymenoptera: Ichneumonidae), Archytas spp. (Diptera: Tachinidae) and Winthenia spp. (Diptera: Tachinidae). 


\section{Introducción}

El cultivo del maíz (Zea mays L.) en el Paraguay, es la segunda en importancia después de la soja, tanto en superficie $(950,000 \mathrm{ha})$ como en producción de granos (4,985,881 Mg). Los departamentos de Alto Paraná, Canindeyú, Itapúa y Caaguazú son los principales productores de maíz, respondiendo por 74,8 \% de la superficie sembrada (DCEA, 2015). Uno de los principales problemas fitosanitarios de este cultivo es el gusano cogollero (S. frugiperda). El método de control más utilizado es la aplicación de productos químicos, siendo muchas veces ineficiente además de encarecer los costos de producción y generan problemas como resistencia de la plaga y contaminación ambiental. En la búsqueda de alternativas de control de esta plaga surge la utilización de parasitoides como una estrategia y oportunidad de proteger al cultivo.

Son registradas la presencia de Chelonus sp., Chelonus cautus Cresson, 1872; Chelonus insularis Cresson, 1865; Cotesia sp.; Cotesia marginiventris Cresson, 1865; Meteorus laphygmae Vierecck, 1913 y Exasticolus sp. (Hymenoptera, Braconidae), Pristomerus spinator Fabricius, 1804; Campoletis flavicincta Ashmead, 1890 y Ophion sp.
(Hymenoptera, Ichneumonidae) y Archytas incertus Giglio-Tos, 1893; Archytas marmoratus Townsend, 1915 y Lespesia archippivora Riley, 1871 (Diptera,Tachinidae) (Dequech et al., 2004; Figueireido et al., 2006 a,b; Cruz et al., 2009; Gutiérrez et al., 2013; Estrada et al., 2013). Ante la falta de informaciones de las especies de parasitoides asociadas al gusano cogollero en Paraguay. El objetivo de este trabajo fue verificar la ocurrencia de parasitoides que actúan como agentes de control biológico natural de larvas de $S$. frugiperda en los principales departamentos productores de maíz.

\section{Materiales y métodos}

\section{Puntos de muestreo}

Se recolectaron larvas de $S$. frugiperda de diferentes cultivos de maíz en los años agrícolas 2014/2015 y 2015/2016, el número de larvas colectadas fue variable. Las recolectas fueron realizadas en cuatro departamentos: Caaguazú, Alto Paraná, Canindeyú e Itapúa que son los principales departamentos productores del maíz en Paraguay. Los datos de temperatura, precipitación, altitud, localidades muestreadas por departamento y número de muestras recolectadas son presentados en la tabla 1.

Tabla 1. Departamento, temperatura, precipitación, altitud, localidades muestreadas y número de muestras realizadas en los años agrícolas 2014/2015 e 2015/2016. Paraguay, 2018.

\begin{tabular}{lrrrr}
\hline Departamento & Temperatura media $\left({ }^{\circ} \mathbf{C}\right)$ & $\begin{array}{r}\text { Precipitación } \\
\text { media }(\mathbf{m m})\end{array}$ & $\begin{array}{r}\text { Altitud } \\
\text { media }(\mathbf{m s n m})\end{array}$ & $\begin{array}{r}\text { Número de localidades } \\
\text { muestreadas y (número } \\
\text { de muestras) }\end{array}$ \\
\hline Canindeyú & 21,92 & 1588 & 268 & $10(1009)$ \\
Caaguazú & 22,06 & 1593 & 236 & $6(1015)$ \\
Alto Paraná & 21,22 & 1630 & 260 & $8(905)$ \\
Itapúa & 21,41 & 1720 & 180 & $10(1115)$ \\
\hline
\end{tabular}

\section{Recolección de larvas}

El número de puntos muestreados (tabla 1), fue variable de acuerdo a la presencia de la plaga. La recolección se realizó en cada lote de manera aleatoria y se recolectaron en promedio 100 larvas de $S$. frugiperda. Las larvas recolectadas en el campo, se identificaron in situ por características morfológicas, luego se colocaron en tubos falcón de $50 \mathrm{~mL}$ y depositados en contenedores del área de Protección Vegetal de la Facultad de Ciencias Agrarias de la Universidad Nacional de Asunción. Una vez en el laboratorio, las larvas de $S$. frugiperda se criaron en tubos de plástico (4,5 cm de diámetro x 7,5 $\mathrm{cm}$ de largo), temperatura de $25 \pm 5^{\circ} \mathrm{C}$, HR $70 \pm 10 \%$ y fotoperiodo de 12 horas. Las larvas fueron alimentadas con hojas de maíz, el cual fue sustituido diariamente hasta que las larvas completaron su ciclo o hasta la emergencia de los parasitoides, las observaciones se realizaron diariamente. Los parasitoides adultos se ubicaron en alcohol 70 \% etiquetados e identificados. 


\section{Identificación de parasitoides}

Los parasitoides se separaron en orden y familia usando las claves taxonómicas de Evans (1964), Achtenberg (1979), Wharton et al. (1997), Brown et al. (2010), López-Martínez et al. (2011) y Rafael et al. (2012). Luego la identificación de género y especie se realizó en el laboratorio de Facultad de Ciencias Exactas y Naturales (FACEN). Las evaluaciones se basaron en la diversidad y frecuencia de las especies de parasitoides emergidos por localidad. Fue calculado el porcentaje de parasitismo según fórmula propuesta por García et al. (2013).

$$
\% \text { de parasitismo }=\frac{\text { № de larvas parasitadas }}{\text { № de larvas útil (total) }} * 100
$$

Donde:

$\mathrm{N}^{0}$ de Larvas Útil: diferencia entre larvas recolectadas y muertas por manejo, fuga o enfermedad.
No de Larvas Parasitadas: solamente a partir de larvas útiles, emergido o no el parasitoide.

\section{Resultados y discusión}

Los levantamientos realizados en los diferentes departamentos, se identificaron siete especies de parasitoides (tabla 2), siendo que las especies recolectadas y porcentaje de parasitismo obtenido variaron, dependiendo de la localidad y departamento. Los porcentajes de parasitoidismo variaron entre 1,85 y $38,46 \%$, ocurriendo parasitismo en todas las localidades. El mayor número de especies recolectadas fue obtenido en los departamentos de Canindeyú y Caaguazú, y la menor diversidad fue observada en Itapúa.

El porcentaje medio de parasitismo en el departamento de Canindeyú fue de 14,80 \% (tabla 3), siendo porcentajes similares encontrados por Murúa et al. (2006) con $15 \%$ de parasitismo en el Noroeste Argentino y de 13,22 \% en Yucatán-México por Delfínet al. (2007).

Tabla 2. Parasitoides de S. frugiperda en los años agrícolas 2014/2015 e 2015/2016, en los departamentos de Alto Paraná, Canindeyú, Itapúa y Caaguazú. 1. Alto Parana 2. Caaguazu 3. Canindeyu 4. Itapúa.

\begin{tabular}{|c|c|c|c|c|c|c|c|}
\hline \multirow[b]{2}{*}{ Orden } & \multirow[b]{2}{*}{ Familia } & \multirow[b]{2}{*}{ Sub familia } & \multirow[b]{2}{*}{ Especie } & \multicolumn{4}{|c|}{ Local } \\
\hline & & & & $\begin{array}{l}\text { Alto } \\
\text { Páraná }\end{array}$ & Caaguazú & Canindeyú & Itapúa \\
\hline \multirow[t]{5}{*}{ Hymenoptera } & Braconidae & Cheloninae & Chelonus sp. & $\mathrm{x}$ & & & \\
\hline & & Homolobinae & Exasticolus fuscicornis & $\mathrm{x}$ & $\mathrm{x}$ & $\mathrm{x}$ & \\
\hline & & & Glyptapanteles militaris & & $\mathrm{x}$ & & \\
\hline & Ichneumonidae & Ophioninae & Ophion sp. & & $\mathrm{x}$ & $\mathrm{x}$ & $\mathrm{x}$ \\
\hline & Bethylidae & & Dissomphalus spp. & & & $\mathrm{x}$ & \\
\hline \multirow[t]{2}{*}{ Diptera } & Tachinidae & Tachininae & Archytas sp. & $\mathrm{x}$ & $\mathrm{x}$ & $\mathrm{x}$ & $\mathrm{x}$ \\
\hline & & Goniinae & Winthenia sp. & $\mathrm{x}$ & $\mathrm{x}$ & $\mathrm{x}$ & $\mathrm{x}$ \\
\hline
\end{tabular}

El mayor porcentaje de parasitismo natural fue de $41,38 \%$ en una recolecta realizada en propiedad de un pequeño productor del distrito de Curuguaty. Este mayor porcentaje pudo deberse al hecho de que este productor tenía un policultivo en su predio y al respecto, Figueiredo et al. (2006a) y Souza (2015), mencionan que una mayor diversidad de especies de parasitoides es observada en policultivos, sistema de producción característico de los pequeños productores, sin embargo en extensas áreas de monocultivos se observa una influencia negativa en relación a la presencia de parasitoides. Las especies de parasitoides en el departamento de Canindeyu fueron: Dissomphalus spp. (Hymenoptera: Bethylidae), Exasticolus fuscicornis Cameron (Hymenoptera: Braconidae), Ophion spp. (Hymenoptera: Ichneumonidae), Archytas spp. (Diptera: Tachinidae) y Winthenia spp. (Diptera: Tachinidae) (tabla 2). Dissomphalus spp., no ha sido reportado como parasitoide de $S$. frugiperda, este ejemplar constituye el primer registro de esta especie asociado al gusano cogollero en Paraguay. Según Redighieri y Azevedo (2006), Dissomphalus es el género más abundante de la familia Bethylidae en bosques tropicales y presentan una distribución amplia en la Mata Atlántica Brasilera. 
Tabla 3. Municipio, coordenadas, número de larvas recolectadas, número de larvas útiles, parasitadas y porcentaje de parasitoidismo natural de $S$. frugiperda en el Departamento de Canindeyu, Paraguay. NLC = número de larva recolectada; NLU = númerfo de larva útiles; $\mathrm{P}=$ porcentaje parasitismo.

\begin{tabular}{|c|c|c|c|c|c|c|}
\hline Recolecta & Municipio & Coordenadas & NLC & NLU & NLP & $\% \mathrm{P}$ \\
\hline 1 & Curuguaty & S - $24^{\circ} 28^{\prime} 56,4^{\prime \prime} \mathrm{W}-055^{\circ} 37^{\prime} 16,4^{\prime \prime}$ & 79 & 53 & 8 & 15,09 \\
\hline 2 & Yvyrarovana & S - $24^{\circ} 24^{\prime} 00,7^{\prime \prime} \mathrm{W}-055^{\circ} 07^{\prime} 35,6^{\prime \prime}$ & 111 & 48 & 17 & 35,42 \\
\hline 3 & Yvyrarovana & S - $24^{\circ} 20^{\prime} 21,1^{\prime \prime} \mathrm{W}-055^{\circ} 07^{\prime} 13,2^{\prime \prime}$ & 105 & 47 & 4 & 8,51 \\
\hline 4 & Yvyrarovana & S - $24^{\circ} 22^{\prime} 16,6^{\prime \prime} \mathrm{W}-054^{\circ} 58^{\prime} 59,9^{\prime \prime}$ & 125 & 78 & 12 & 15,38 \\
\hline 5 & Yvyrarovana & S - $24^{\circ} 22^{\prime} 11,4^{\prime \prime} \mathrm{W}-055^{\circ} 07^{\prime} 16,7^{\prime \prime}$ & 75 & 59 & 2 & 3,39 \\
\hline 6 & Yvyrarovana & S - $24^{\circ} 18^{\prime} 12,4^{\prime \prime} \mathrm{W}-054^{\circ} 58^{\prime} 34,5^{\prime \prime}$ & 95 & 36 & 5 & 13,89 \\
\hline 7 & Curuguaty & S $-24^{\circ} 15^{\prime} 44,3^{\prime \prime} \mathrm{W}-055^{\circ} 43^{\prime} 53,5^{\prime \prime}$ & 90 & 54 & 1 & 1,85 \\
\hline 8 & Curuguaty & S $-24^{\circ} 15^{\prime} 38,0^{\prime \prime} \mathrm{W}-055^{\circ} 43^{\prime} 50,7^{\prime \prime}$ & 115 & 70 & 9 & 12,86 \\
\hline 9 & Curuguaty & S $-24^{\circ} 15^{\prime} 27,6^{\prime \prime} \mathrm{W}-055^{\circ} 43^{\prime} 53,7^{\prime \prime}$ & 114 & 29 & 12 & 41,38 \\
\hline 10 & Curuguaty & S $-24^{\circ} 15^{\prime} 35,0^{\prime \prime} \mathrm{W}-055^{\circ} 44^{\prime} 08,2^{\prime \prime}$ & 100 & 80 & 12 & 15,00 \\
\hline Total & & & 1009 & 554 & 82 & 14,80 \\
\hline
\end{tabular}

En el Departamento de Caaguazú (tabla 4), el porcentaje medio de parasitismo fue de $20,25 \%$, siendo semejante a los encontrados por Dequech et al. (2004), Murúa et al. (2006), Murúa et al. (2009), Silva et al. (2011), Ordóñez et al. (2015a) y Ordóñez et al. (2015b) que obtuvieron parasitismo de 22,$01 ; 19,94 ; 18,93 ; 23 ; 18,20$ y $22,08 \%$ respectivamente, en países de América. Sin embargo, es considerado bajo cuando comparado con los trabajos de García et al. (2013), que mencionan parasitismo de 62,40 $\%$ y de $48,46 \%$ respectivamente en México. El parasitismo varío entre $15,32 \%$ en el municipio de 3 de febrero hasta $36,45 \%$ en la localidad de Dr. J. M. Frutos, pudiendo ser considerado como aceptable ya que en la zona es utilizada gran cantidad de productos químicos no selectivos. Del total de 152 parasitoides emergidos, con cinco especies identificadas (tabla 2), Glyptapanteles militaris fue la más común en las localidades de repatriación y 3 de febrero, a su vez Archytas sp. fue la más abundante en Yhú y Campo 9, mientras en Dr. J.M. Frutos, la especie con mayor número de parasitoides emergidos fue Winthenia sp. y en Caaguazú la más abundante fue Ophion sp.

En el departamento de Alto Paraná hubo un parasitismo natural de 16,73\% (tabla 5), variando entre 2,56\% (Itakyry) y 38,46\%. El valor medio podría ser considerado bajo al comparar como los resultados presentados por Dequech et al. (2004), Pérez (2008) y Ordoñez et al. (2015a). Para las localidades de Alto Paraná la especie más abundante fue Exasticolus fuscicornis, mientras la menos abundante fue Chelonus sp. (tabla 1).

Tabla 4. Municipio, coordenadas, número de larvas recolectadas, larvas útiles, parasitadas y porcentaje de parasitidoismo natural de $S$. frugiperda en el Departamento de Caaguazú, Paraguay. NLC= Número de larva recolectada; NLU = Número de larva útiles; $\% \mathrm{P}=$ porcentaje parasitismo.

\begin{tabular}{|c|c|c|c|c|c|c|}
\hline Recolecta & Municipio & Coordenadas & NLC & NLU & NLP & $\% \mathrm{P}$ \\
\hline 1 & Caaguazú & S - $25^{\circ} 27^{\prime} 20,60^{\prime \prime W}-055^{\circ} 59^{\prime \prime} 00,83^{\prime \prime}$ & 190 & 138 & 24 & 17,39 \\
\hline 2 & Dr. Frutos & S - $25^{\circ} 19^{\prime} 52,02^{\prime \prime} \mathrm{W}-055^{\circ} 51^{\prime \prime} 12,48^{\prime \prime}$ & 225 & 96 & 35 & 36,45 \\
\hline 3 & Yhú & S - $25^{\circ} 17^{\prime} 08,60^{\prime \prime}$ W $-055^{\circ} 58^{\prime} 32,13^{\prime \prime}$ & 215 & 96 & 17 & 17,70 \\
\hline 4 & Campo 9 & S - $25^{\circ} 22^{\prime} 53,14^{\prime \prime}$ W $-055^{\circ} 40^{\prime} 10,91^{\prime \prime}$ & 120 & 56 & 9 & 16,07 \\
\hline 5 & Repatriación & S - $25^{\circ} 32^{\prime} 07,51^{\prime \prime} \mathrm{W}-055^{\circ} 40^{\prime} 10,91^{\prime \prime}$ & 55 & 30 & 6 & 20 \\
\hline 6 & 3 de febrero & S $-55^{\circ} 13^{\prime} 40,47^{\prime}$ W $-055^{\circ} 46^{\prime} 56,56^{\prime \prime}$ & 210 & 137 & 21 & 15,32 \\
\hline Total & & & 1015 & 553 & 112 & 20,25 \\
\hline
\end{tabular}


Tabla 5. Municipio, número de larvas colectadas, larvas útiles, parasitadas y porcentaje de parasitoidismo natural de $S$. frugiperda en el Departamento de Alto Paraná Paraguay. NLC = Número de larva colectada; NLU = Número de larva útiles; \% P = Porcentaje parasitismo.

\begin{tabular}{|c|c|c|c|c|c|c|}
\hline Recolecta & Municipio & Coordenadas & NLC & NLU & NLP & $\% \mathbf{P}$ \\
\hline 1 & Dr. Mallorquin & $\mathrm{S}-25^{\circ} 27^{\prime} 19^{\prime \prime} \mathrm{W}-55^{\circ} 13^{\prime} 05^{\prime \prime}$ & 38 & 26 & 6 & 7,69 \\
\hline 2 & Yguazú & $\mathrm{S}-25^{\circ} 22^{\prime} 40^{\prime \prime} \mathrm{W}-54^{\circ} 55^{\prime} 29^{\prime \prime}$ & 15 & 6 & 5 & 6,41 \\
\hline 3 & Hernandarias & $\mathrm{S}-25^{\circ} 16^{\prime} 03^{\prime \prime} \mathrm{W}-54^{\circ} 51^{\prime} 13^{\prime \prime}$ & 102 & 45 & 11 & 14,1 \\
\hline 4 & Itakyry & S - $25^{\circ} 01^{\prime} 24^{\prime \prime} \mathrm{W}-55^{\circ} 00^{\prime} 09^{\prime \prime}$ & 55 & 38 & 2 & 2,56 \\
\hline 5 & Mbarakayú & $\mathrm{S}-25^{\circ} 05^{\prime} 32^{\prime \prime} \mathrm{W}-54^{\circ} 57^{\prime} 26^{\prime \prime}$ & 164 & 86 & 10 & 12,82 \\
\hline 6 & Minga Porã & S - $24^{\circ} 53^{\prime} 42^{\prime \prime} \mathrm{W}-54^{\circ} 56^{\prime} 35^{\prime \prime}$ & 285 & 130 & 30 & 38,46 \\
\hline 7 & San Alberto & $\mathrm{S}-24^{\circ} 56^{\prime} 15^{\prime \prime} \mathrm{W}-54^{\circ} 56^{\prime} 47^{\prime \prime}$ & 84 & 41 & 5 & 6,41 \\
\hline 8 & Santa Fé & S $-25^{\circ} 16^{\prime} 53^{\prime \prime} \mathrm{W}-54^{\circ} 43^{\prime} 23^{\prime \prime}$ & 162 & 100 & 9 & 11,53 \\
\hline Total & & & 905 & 472 & 78 & 16,73 \\
\hline
\end{tabular}

El porcentaje medio en el departamento de Itapúa fue de 19,3 \% (tabla 6), siendo el valor similar a los presentados por Molina et al. (2004), Silva et al. (2011) y García et al. (2013). El porcentaje más bajo de parasitismo fue obtenido en el municipio de Pirapó $(5,50 \%)$ y Natalio (5,90 \%), mientras que el valor más elevado fue obtenido en el municipio de Edelira (37,50 \%), los valores bajos pueden estar relacionados con el uso de químicos no selectivos aplicados continuamente en la zona. En las localidades monitoreadas, la especie más abundante fue Whinthenia sp. seguida de Archytas sp., mientras que la especie menos abundante fue Ophion sp. Estos resultados coincide con los trabajos de Murúa et al. (2006), los cuales mencionan a la familia Tachinidae como los más abundantes en la Argentina.

Tabla 6. Municipio, número de larvas colectadas, larvas útiles, parasitadas y porcentaje de parasitoidismo natural de $S$. frugiperda en el Departamento de Itapúa. Paraguay. $\mathrm{NLC}=$ Número de larva recolectada; NLU = Número de larva útiles; \% P= Porcentaje parasitismo.

\begin{tabular}{|c|c|c|c|c|c|c|}
\hline Recolecta & Município & Coordenadas & NLC & NLU & NLP & $\% \mathrm{P}$ \\
\hline 1 & Pirapó & 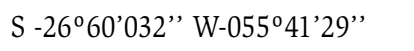 & 73 & 55 & 3 & 5,45 \\
\hline 2 & Capitán Miranda & S -2702'972”'W- $055^{\circ} 57^{\prime} 199^{\prime \prime}$ & 29 & 18 & 4 & 22,22 \\
\hline 3 & Fram & 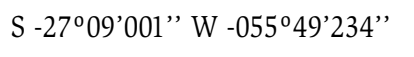 & 60 & 40 & 10 & 25 \\
\hline 4 & La Paz & S $-27^{\circ} 04^{\prime} 890^{\prime \prime} \mathrm{W}-055^{\circ} 30^{\prime} 742^{\prime \prime}$ & 46 & 23 & 3 & 13,04 \\
\hline 5 & Obligado & S - $27^{\circ} 04^{\prime} 27^{\prime \prime} \mathrm{W}-055^{\circ} 38^{\prime} 18^{\prime \prime}$ & 29 & 17 & 4 & 23,53 \\
\hline 6 & Hohenau & S - $27^{\circ} 07^{\prime} 221^{\prime \prime} \mathrm{W}-055^{\circ} 51^{\prime} 208^{\prime \prime}$ & 77 & 39 & 5 & 12,82 \\
\hline 7 & Capitán Meza & S - $26^{\circ} 50^{\prime} 06^{\prime \prime} \mathrm{W}-055^{\circ} 30^{\prime} 502^{\prime \prime}$ & 37 & 27 & 5 & 18,52 \\
\hline 8 & María Auxiliadora & S - $26^{\circ} 46^{\prime} 20.17 \mathrm{~W}-055^{\circ} 16^{\prime} 34^{\prime \prime}$ & 318 & 231 & 27 & 14,33 \\
\hline 9 & Edelira & S - $26^{\circ} 37^{\prime} 15.0^{\prime \prime} \mathrm{W}-055^{\circ} 19^{\prime} 50^{\prime \prime}$ & 39 & 24 & 9 & 37,50 \\
\hline 10 & Natalio & S - $26^{\circ} 45^{\prime \prime} 35^{\prime \prime} \mathrm{W}-055^{\circ} 10^{\prime} 20^{\prime \prime}$ & 407 & 272 & 16 & 5,88 \\
\hline Total & & & 1115 & 746 & 86 & 11,53 \\
\hline
\end{tabular}


Las especies Chelonus sp., Exasticolus fuscicornis, Glyptapanteles militaris; Ophion sp.; Archytas sp. y Winthenia sp. encontradas en esta investigación, ya habían sido reportadas como parasitoides de $S$. frugiperda y otros noctuideos (Cave, 1993; Dequech et al., 2004; Estrada et al., 2013; Gutiérrez et al., 2013), en países como Brasil, México, Honduras, Cuba. La excepción la constituye la especie Dissomphalus spp., siendo este el primer registro de parasitoidismo en larvas de S. frugiperda.

Cabe resaltar que estas especies han sido reportadas como parasitoides naturales, sin embargo, no son producidos a gran escala, siendo así son necesarios estudios básicos para determinar su potencial como controladores biológicos como base para una producción masa.

\section{Conclusiones}

Con base a los resultados en los distintos departamentos donde fueron realizadas las recolectas, se puede concluir que la ocurrencia natural de parasitoides en el cultivo de maíz, se constituyen en importantes componentes de la regulación de la población de plagas, debiendo ser garantizada la conservación de las mismas, a través de manejos ecológicos.

Fueron encontradas siete especies de parasitoides de $S$. frugiperda representados por las siguientes cuatro familias y sus respectivas especies, Braconidae: Chelonus sp., Exasticolus fuscicornis, Glyptapanteles militaris; Ichneumonidae: Ophion sp.; Bethylidae: Dissomphalus spp. y Tachinidae: Archytas sp. y Winthenia sp.

El parasitismo natural varió entre los departamentos, siendo de 14,8 \%; 20,25\%; 16,73\% y 11,53 \% para los departamentos de Canindeyú, Caaguazú, Alto Paraná e Itapúa, respectivamente.

\section{Referencias}

Achterneng, C. 1979. A revision of the subfamily Zelinae auct. (Hymenoptera, Braconidae). Tijdschrift voor Entomologie 122(7): 241-479.

Brown, B., Borkent, A., Cumming, J., Wood, N., Woodley, N. y Zumbado, A. 2010. Manual of Central American Diptera. Resench Press, Otawa.

Cave, R. 1993. Parasitoides larvales y rurales dé Spodoptera 135 frugiperda (Smith) (Lepidoptera:Noctuidae) en Centro
América con una clave para las especies encontradas en Honduras. Revista CEIBA 34(1): 33 - 56.

Cruz, I., Figueireido, M., Silva, R., Del Sarto, M. y PenteadoDias, A. 2009. Monitoramento de parasitoides de lagartas de Spodoptera frugiperda (J. E. Smith) (Lepidoptera: Noctuidae) em Municipios de Minas Gerais, Brasil. Siete Lagunas. EMBRAPA Maíz y Sorgo, Siete Lagunas.

DCEA (Dirección de Censos y Estadísticas Agropecuarias, Py). 2015. Estimación de superficie, producción y rendimiento de cultivos agrícolas del Paraguay. Ministerio de Agricultura y Ganadería._URL. Disponible en:_http://www.mag.gov.py/ Censo/SINTESIS\% 202015.pdf. Consultado: 12 de septiembre de 2015.

Dequech, S., da Silva, R. y Fiuza, L. 2004. Ocorrência de parasitóides de Spodoptera frugiperda (J. E. Smith) (Lepidoptera, Noctuidae) en lavouras de milho en Cachoeirinha, RS. Ciência Rural 34(4): 1235 - 1237.

Delfín-González, H., Bojorquez-Acevedo, M. y ManriqueSaide, P. 2007. Parasitoids of fall armyworm (Lepidoptera: Noctuidae) from a traditional maize crop in the Mexican state of Yucatan. Florida Entomologist 90(4): 759-761.

Estrada- Virgen, O., Cambero-Campos, J., Robles-Bermudez, A., Rios-Velasco, C., Carbajar-Casola, C., Isiorda-Aquino, N. y Ruiz-Cancino, E. 2013. Parasitoides y entomopatógenos nativos asociados al gusano cogollero Spodoptera frugiperda (Lepidoptera: Noctuidae) en Nayarit, México. Southwestern Entomologist 38 (2): 339-344.

Evans, H. 1964. A sinapsis of the American Bethylidae (Hymenoptera, Aculeata). Bulletin of the Museum of Comparative Zoology 132 (1):1 - 222.

Figueiredo, M., Martins- Dias, A. y Cruz, I. 2006a. Associação entre inimigos naturais e Spodoptera frugiperda (J. E. Smith 1797) (Lepidoptera: Noctuidae) na cultura do milho. Revista Brasileira de milho e sorgo 5(3): 340 -350.

Figueiredo, M., Martins- Dias, A. y Cruz, I. 2006 b. Relação entre a lagarta-do-cartucho e seus agentes de controle biológico natural na produção de milho. Pesquisa agropecuaria brasileira 41(12):1693-1698.

García, C. González, M. y González, A. 2013. Parasitismo natural de Braconidae e Ichneumonidae (Hymenoptera) sobre Spodoptera frugiperda (Lepidoptera: Noctuidae). Revista Colombiana de Entomología 39(2): 211-215.

Gutiérrez, A., Robles, A., Cambero, J., Santillan, C., Ortiz, M., Coronado, J. y Campos, M. 2013. Parasitoide de Spodoptera 
frugiperda (Lepidóptera: Noctuidae) encontrados en Nayarit, México. Southwestern Entomologist 40(3): 555-564.

López, V., Saavedra, M., Delfin, H., Figueroa, J. y García, M. 2011. A new species of the genus Exasticolus van Achterbeng (Hymenoptera: Braconidae: Homolobinae) from Mexico. Studies on Neotropical Fauna and Environment 46(1):59-62.

Molina, J., Carpenter, J., Lezama, R., Foster, J. y González, M. 2004. Natural distribution of Hymenopteran parasitoids of Spodoptera frugiperda (Lepidoptera: Noctuidae) larvae in México. Florida Entomologist 87(4): 461-472.

Murúa, G., Molina, J. y Coviella, C. 2006. Population dynamics of the fall armyworm, Spodoptera frugiperda (Lepidoptera: Noctuidae) and its parasitoids in northwestern Argentina. Florida Entomologist 89(2): 175-182.

Murúa, G., Molina, J. y Fidalgo, P. 2009. Natural distribution of parasitoids of larvae of the fall armyworm, Spodoptera frugiperda, in Argentina. Journal of Insect Science 9(20):1-17. 2009.

Ordoñez, M., Ríos, C., Berlanga, D., Acosta, C., Salas, M. y Cambero, J. 2005 a. Occurrence of natural enemies of Spodoptera frugiperda (Lepidoptera: Noctuidae) in Chihuahua, México (en línea). Florida Entomologist 98(3): $843-847$.

Ordóñez, M., Bustillos, J., Loya, J., Ríos, C., y Jacobo, J. 2015 b. Parasitoides de Spodoptera frugiperda (J. E. Smith)
(Lepidoptera: Noctuidae) en Chihuahua, México. Métodos en ecología y sistemática. 10(1): 67-78.

Pérez, E. 2008. Control biológico de Spodoptera frugiperda Smith en maíz. INISAV: Habana.

Rafael, J., Melo, G., de Carvalho, C., Constantino, R. 2012. Insetos do Brasil: Diversidade e Taxonomia. Holos Editorial, Ribeirão Preto.

Redighieri, E, y Azevedo, C. 2006. Fauna de Dissomphalus Ashmead (Hymenoptera, Bethylidae) da Mata Atlântica Brasileira, com descrição de 23 espécies novas. Revista Brasileira de Entomologia 50(3): 297-334.

Silva, F., Cruz, I., Figueiredo, M., Costa, M., Redoan, A., y Morato, J. 2011. Dinâmica populacional de parasitoides de Spodoptera frugiperda (J. E. Smith) (Lepidoptera: Noctuidae) en milho (Zea mays L.) cultivado no sistena orgânico de produção. Cadernos de Agroecología 6(2): 1-5.

Souza, A. 2015. Diversidade de himenópteros parasitoides en agroecossistenas. Maestria em Zoologia, Universidad de Brasilia, Brasilia, Brasil.

Wharton, R., Marsh, P. y Sharkey, M. 1997. Manual of the new world genera of the Family Braconidae (Hymenoptera). Madison: USA.

Citar como: Cabral-Antúnez, C.C., Garcete, B., Montiel-Cáceres, R.I., Gonzalez-Vega, A.B., Cárdenas S.R., Armoa, N. y María Ramírez de López, M.B. 2018. Parasitismo Natural de Spodoptera frugiperda (Smith) (Lepidoptera: Noctuidae), en cuatro departamentos de Paraguay. Intropica 13(2): 130-136. DOI: http://dx.doi.org/ 10.21676/23897864.2655. 